\title{
Evaluación de la satisfacción de las familias y/o acompañantes de los pacientes atendidos en la unidad de cuidado intensivo y cuidado coronario de una institución de cuarto nivel de complejidad de la ciudad de Medellín
}

Satisfaction evaluation of families and/or companions of patients treated in the intensive care and the coronary care unit of a fourth level complexity institution in Medellin

Avaliação da satisfação das famílias e / ou acompanhantes de pacientes tratados na unidade de terapia intensiva e de cuidados coronários de uma instituição de quarto nível de complexidade da cidade de Medellín

Ana María Cadavid González ${ }^{\circledR}$, Daniela Guerra Sánchez ${ }^{\star}$, Gladys Del Toro Morales ${ }^{3}$

Fecha correspondencia:

Recibido: noviembre 10 de 2015. Revisado: diciembre 04 de 2015. Aceptado: febrero 24 de 2016.

\section{Forma de citar:}

Cadavid-González AM, Guerra D, Del Toro M. Evaluación de la satisfacción de las familias y/0 acompañantes de los pacientes atendidos en la unidad de cuidado intensivo y cuidado coronario de una institución de cuarto nivel de complejidad de la ciudad de Medellín . Rev CES Salud Pública. 2016; 7(1): 25-36.

Open access

(c) Derecho de autor

Licencia creative commons

Ética de publicaciones

Revisión por pares

Comparte

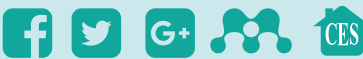

\section{Resumen}

Introducción: aplicar una encuesta de satisfacción a las familias o acompañantes de los pacientes que egresan de la unidad de cuidado intensivo y cuidado coronario de una institución de cuarto nivel de complejidad del 16 de julio al 30 de agosto de 2014. Materiales y métodos: se realizó un estudio descriptivo retrospectivo sobre la satisfacción de la familia y/o acompañantes de los pacientes al egreso de la unidad de cuidados intensivos (UCI) y cuidados coronarios (UCC) en una institución de cuarto nivel de complejidad de la ciudad de Medellín del 16 de julio al 30 de agosto de 2014. Se empleó la encuesta de "satisfacción a los familiares de pacientes críticos" aplicado en el 2006 en el servicio de medicina intensiva del Hospital Universitario Insular de Gran Canaria, Las Palmas España. Resultados: se evaluaron cuatro conceptos como la información brindada sobre el estado del paciente, la terminología utilizada y la explicación sobre los equipos médicos utilizados; los cuidados recibidos por el paciente por parte del personal sanitario (médicos, enfermeras, auxiliares); el trato por parte del personal médico incluyendo preguntas sobre las necesidades especiales a los acompañantes y si consideran que en caso de alguna urgencia le llamaran a su casa y por último la comodidad de la sala de espera. Conclusiones: los familiares y/o acompañantes de los pacientes encuestados se encuentran satisfechos con los cuatro conceptos evaluados aunque se debe analizar en la institución aspectos tan importantes como la seguridad de que los llamaran en caso de urgencia y la comunicación sobre la atención del paciente ya que estas pueden mejorarse.

Palabras clave: encuestas de salud, satisfacción personal, asistencia del enfermo crítico, relaciones profesional-familia. 
Enero - Junio 2016 - Pág 26

Gestión por Open Journal System

ISSN 2145-9932

Sobre los autores:

1. Médica general. Especialista en auditoría en salud.

2. Enfermera profesional. Especialista en auditoría en salud.

3. Enfermera profesional. Especialista en auditoría en salud.

\section{Abstract}

Introduction: to apply a satisfaction survey to the families and/or companions of the patients discharged from the intensive care and coronary care unit of a fourth level complexity institution from July 16th to August 30th. Methods: a retrospective descriptive study of patient's family and / or companions satisfaction at discharge from the intensive care unit and coronary care unit was conducted from July 16 th to August 30th of 2014. The Survey used was "satisfacción a los familiares de pacientes críticos" which was applied in 2006 in the intensive care unit of the Hospital Universitario Insular in Gran Canaria, Las Palmas - Spain. Results: four concepts were evaluated in the survey such as the information provided about the patient's condition, the terminology used and the explanation of the medical equipment used; care received by the patient by the medical staff (doctors, nurses, assistants); treatment by medical staff including questions to the companions about their personal needs and whether they feel that in case of an emergency they will be called home and finally the comfort of the waiting room. Conclusions: the families and companions of the patients included in the survey were satisfied with the four evaluated concepts. The institution must analyze critical aspects such as the confidence that the will be called home in case of an emergency and the communication about the care of the patient that can be improved.

Keywords: Health surveys, personal satisfaction, critical care, professional-family relations.

\section{Resumo}

Introdução: aplicar um famílias pesquisa de satisfação ou acompanhantes de pacientes que deixam a unidade de terapia intensiva e de cuidados coronários de uma instituição de quarto nível de complexidade do 16 julho-30 agosto de 2014. Método: estudo retrospectivo sobre a satisfação da família e / ou acompanhantes de pacientes no momento da alta da unidade de terapia intensiva (UTI) e de cuidados coronários (UCC) foi realizada em uma instituição de quarto nível de complexidade da Cidade Medellin a partir de 16 julho - 30 agosto de 2014. o levantamento de "satisfação às famílias dos pacientes criticamente doentes" aplicadas em 2006 na unidade de terapia intensiva do Hospital Universitário Insular de Gran Canaria, foi usada Las Palmas Espanha. Resultados: quatro conceitos como a informação prestada sobre a condição do paciente foram avaliados, a terminologia utilizada ea explicação de equipamentos médicos utilizados; o atendimento recebido pelo paciente pela equipe médica (médicos, enfermeiros, assistentes); tratamento pelo pessoal médico, incluindo perguntas sobre as necessidades especiais de acompanhamento e, se eles sentem uma certa urgência, se você ligar para casa e, finalmente, o conforto da sala de espera. Conclusões: família e / ou acompanhantes de pacientes pesquisados estão satisfeitos com os quatro conceitos avaliadas, mas devem ser analisadas na instituição questões tão importantes como a segurança que ligar em caso de emergência e comunicação no atendimento ao paciente e estes podem ser melhoradas.

Palavras-chave: Inquéritos Epidemiológicos, Satisfação Pessoal, Cuidados Críticos, Relações Profissional-Família 


\section{Introducción}

El concepto de satisfacción ha venido evolucionando con el transcurso de los días planteándose al principio en 1977 por Hunt como una "Evaluación que analiza si una experiencia de consumo es al menos tan buena como se esperaba" (1) y en 1996 por Oliver como el "Juicio del resultado que un producto o servicio ofrece para un nivel suficiente de realización en el consumo" (1). Todas estas apuntan a que sea el logro de un objetivo de acuerdo a un referente o estándar.

Recientemente el significado de satisfacción ha tenido otra connotación la cual es explicada por Philip Kotler quien la define como "El nivel del estado de animo de una persona que resulta de comparar el rendimiento percibido de un producto o servicio con sus expectativas" (1), dándose a entender como la percepción y evaluación que realiza el usuario frente a un servicio y que depende si respondió a sus necesidades y expectativas.

Este concepto se volvió un referente en la prestación del servicio a los usuarios teniendo como base la normatividad Colombiana como la Ley 100 de 1993 en su artículo 199, el Decreto 2309 de 2002, la Circular Externa 000030 de 2006, que establecen la calidad en la atención debe estar basada en actividades encaminadas a garantizar los servicios de salud accesibles y equitativos con profesionales óptimos y teniendo en cuenta los recursos disponibles, logrando la satisfacción del usuario con la atención recibida.

Hasta finales de la década de los 70 , tan solo se consideraba importante la repercusión que el ingreso en una unidad de cuidado intensivo (UCI) o unidad de cuidado coronario (UCC) podía tener sobre el paciente. No fue hasta 1976, tras la publicación de la tesis de Molter, cuando se comenzó a dedicar especial atención a ese colectivo, hasta entonces ignorado, formado por los familiares de los ingresados en $\mathrm{UCl}(\underline{2})$.

Hampe en 1975, fue la primera investigadora que evaluó las necesidades de duelo de las esposas de pacientes hospitalizados encuestándolas y determinando su nivel de satisfacción frente a la realidad del paciente encontrando como fundamental al momento de la evaluación el poder estar con la persona que estaba muriendo, la necesidad de ayudarle y de estar seguras de que esa persona estaban tan cómoda física y emocionalmente como era posible (2). Desde el punto de vista personal, se describía la importancia de la información y el apoyo del personal a cargo del paciente. Los resultados demostraron que ninguna de las esposas quedó satisfecha, la información brindada solo fue suficiente para la mitad de los casos y solo 40 - $45 \%$ de las esposas reportaron satisfacción. Estos estudios influenciaron y motivaron posteriores investigaciones en familiares de pacientes en cuidado crítico (ㅁ).

Basado en las experiencias de Hampe, Molter desarrollo en 1979 el Critical care Family Needs Inventory (CCNFI) que consistía en una lista de 45 necesidades que las familias podían calificar en una escala de 4 (4); en 1983, Molter y Leske crearon la versión definitiva del cuestionario CCFNI, con algunas modificaciones sobre el original $(\underline{5}, \underline{6})$. El cuestionario consta de una lista de 45 ítems que miden las necesidades percibidas por los miembros de la familia del paciente ingresado en UCl. Los ítems se valoran en una escala tipo likert que va de 1 (no es muy importante) a 4 (es muy importante). La puntuación total se obtiene sumando las respuestas y el total va de 45 a 180 indicando a mayor puntuación más necesidades percibidas. El cuestionario está estructurado en cinco factores que evalúan la necesidad de apoyo, el confort, la información, la proximidad y la seguridad (ㅁ). 
Existe también El Needs Met Inventory (NMI) de Warren (8)), el cual mide las necesidades que han sido satisfechas durante la estancia en UCl. Este cuestionario consta de 45 ítems que se corresponden con las 45 afirmaciones del CCFNI. Del mismo, existe también una versión breve creada en $1993(\underline{8}, \underline{9})$ la cual redujo la lista de ítems de 45 a 14 ítems tipo likert, con los que se pretendía medir la capacidad de los profesionales para satisfacer las necesidades de los familiares del paciente de UCl. Posteriormente, Johnson y col (10) se encargaron de validar esta versión breve encontrando 4 dimensiones: actitud, comunicación, consuelo y aislamiento. Los resultados sugieren que las principales necesidades de los familiares son la información oportuna, honesta y fácilmente entendible, la seguridad y la cercanía con el paciente (11).

El ingreso a hospital suele causar grandes niveles de estrés tanto para el paciente como para sus familiares (12). Este sentimiento es especialmente importante cuando los pacientes son atendidos en una Unidad de Cuidados Intensivos o cuidados coronarios, dadas las connotaciones negativas que supone el ingreso en esta unidad, en la que se presupone la gravedad del paciente, con todos los temores que este hecho puede conllevar; la admisión a UCI o UCC genera emociones fuertes tales como shock, negación, ira, desespero, culpa, miedo, entre otras.

Las personas involucradas en la atención del paciente crítico son el médico, la enfermera, el personal de apoyo y la familia del paciente. Parece haber un consenso general que explica que el involucrar los familiares en el proceso de atención es importante y tiene efectos positivos tanto en el paciente como en la familia. Investigaciones sugieren que los familiares no reciben suficiente atención por razones realmente poco claras (13), incluso algunos estudios siguieren que el personal de enfermería a menudo evalúa erróneamente las necesidades de las familias (14).

Estudios han indicado que los miembros de la familia pueden ver la comunicación con el médico tratante incluso más importante que sus habilidades clínicas $(\underline{15}, 16)$; sin embargo, se ha demostrado que solo la mitad de las familias de pacientes en $\mathrm{UCl}$ o UCE comprenden suficientemente el diagnóstico, tratamiento y pronostico después de las reuniones con los médicos (17). Por otra parte, las actitudes del personal médico frente a la importancia de la comunicación varían gradualmente (18), debido a estas preocupaciones, recientes recomendaciones instan a los clínicos para mejorar sus habilidades de comunicación y mejorar la relación con las familias $(\underline{18}, \underline{19})$.

La severidad de la enfermedad del paciente y su estado de consciencia hacen difícil la interpretación de la percepción de satisfacción del paciente crítico (20). Usualmente su estado de salud se encuentra tan comprometido que el mismo paciente no está en condiciones de calificar la calidad de la atención prestada e incluso en ocasiones la medicación requerida para su tratamiento altera de tal forma su estado de consciencia que el paciente no recuerda momentos de su estancia en la unidad. Basados en lo anterior, las encuestas de satisfacción aplicadas a los pacientes son inadecuadas.

La satisfacción de la atención debe medirse entonces según la percepción de los miembros de la familia quienes determinan si el paciente recibió atención con calidad independiente del resultado clínico (18). Los familiares del paciente son la fuente más importante de apoyo y conexión entre el paciente y el equipo médico (21).

Están descritos diferentes predictores de satisfacción de la familia como son: relación de enfermeras contra pacientes de 3 a 1 o menos, información provista por el 
médico, información dada por la familia, conocimiento del rol de cada actor (enfermera, medico, familiar), relación de la familia con el médico tratante del paciente, tiempo de visita deseado versus permitido.

Conocer las necesidades de los familiares de los pacientes es una actividad esencial del personal de las UCI o UCE (22). Si bien es cierto que la no restricción de los horarios de visita puede ser beneficioso para el paciente y su familia, los horarios restringidos siguen siendo comunes en las $\mathrm{UCI}(\underline{20})$. El personal de enfermería usualmente prefiere horarios de visita restringidos ya que el exceso de estos se presume como un detrimento a la salud del paciente por tener menos tiempo de descanso y además por la organización del cuidado del mismo ya que probablemente se interrumpiría el que hacer de enfermería (23).

Es por esto que las instituciones han implementado herramientas e instrumentos como los procesos de quejas, reclamos, sugerencias, encuestas y entrevistas, las cuales contribuyen al resultado de este atributo de calidad para conocer la percepción de sus usuarios frente a cada uno de sus servicios, generando no solo calidad si no también lealtad, competitividad y fiabilidad.

\section{Materiales y métodos}

Se realizó un estudio descriptivo retrospectivo sobre la satisfacción de la familia y/o acompañantes de los pacientes al egreso de la unidad de cuidados intensivos y unidad de cuidados coronarios en una institución de cuarto nivel de complejidad de la ciudad de Medellín durante el periodo comprendido del 16 de junio al 30 de agosto de 2014.

Se utilizó la encuesta de "Satisfacción a los familiares de pacientes críticos" aplicado en el 2006 en el servicio de medicina intensiva del Hospital Universitario Insular de Gran Canaria, Las Palmas España. Para la implementación de esta se contó con autorización del autor, el médico especialista en medicina intensiva Luciano Santana Cabrera.

La muestra estuvo conformada por 316 encuestas realizadas a pacientes, familiares y/o acompañantes y corresponde a todos los pacientes que ingresaron a las unidades de cuidados intensivos y cuidados coronarios en el período comprendido entre el 16 de junio y el 30 de agosto del 2014. Del total de encuesta, 208 fueron para pacientes de la unidad coronaria y 108 de la unidad de cuidados intensivos. Se excluyeron las encuestas donde no respondieron todas las preguntas.

La base de datos se obtuvo en formato SAV y se efectuó el análisis de los mismos en el programa SPSS $21{ }^{\circledR}$ (Licencia Universidad CES). Se calcularon proporciones para las variables cualitativas.

Esta investigación fue avalada por el Comité Operativo de Investigaciones de la Facultad de Medicina de la Universidad CES y se tomó consentimiento informado a los pacientes y/o familiares o acompañantes del paciente.

\section{Resultados}

Del 16 de junio al 30 de agosto se encuestaron 306 familias y/o pacientes ingresados a las unidades de cuidado intensivo y cuidado coronario. Doscientos ocho (208) encuestas fueron realizadas en la unidad coronaria y 108 en la unidad de cuidado 
intensivo. Seis familiares se negaron a contestar la encuesta y firmaron su disentimiento en el consentimiento informado.

Las características demográficas los pacientes se observan en la tabla 1. Diez encuestas de la unidad coronaria (3.2 \% del total de encuestados) fueron respondidas por el propio paciente ya que no contaban con acompañante y durante su estancia nunca tuvieron alteración del estado de consciencia que limitara su concepto o percepción de la satisfacción.

Tabla 1. Características demográficas de los pacientes hospitalizados en la UCl y UCC

\begin{tabular}{lccc}
\hline & Global & UCI & UCC \\
\hline Total encuestas & 316 & 108 & 208 \\
Edad & 15 a 94 años & 15 a 88 años & 16 a 94 años \\
Genero & Hombres: $179(59.1 \%)$ & Hombres: $63(59.4 \%)$ & Hombres: $116(58.9 \%)$ \\
Días estancia & 1 a 59 días & 1 a 59 días 20 días \\
& 2 hora: $7.6 \%$ & Menor de 5 días: $91 \%$ & Menor de 5 días: $92.3 \%$ \\
Tiempo de visita global $12.5 \%$ & 3 horas o más: $79.9 \%$ & Más de 3 horas: $71(68.9 \%)$ & Más de 3 horas: 171 (85.5 \%) \\
Tiempo de visita por unidad & &
\end{tabular}

Los cinco principales diagnósticos de los pacientes internados en cada unidad se evidencian en la figura 1 para la unidad de cuidado intensivo y la figura 2 para la unidad de cuidados coronarios.

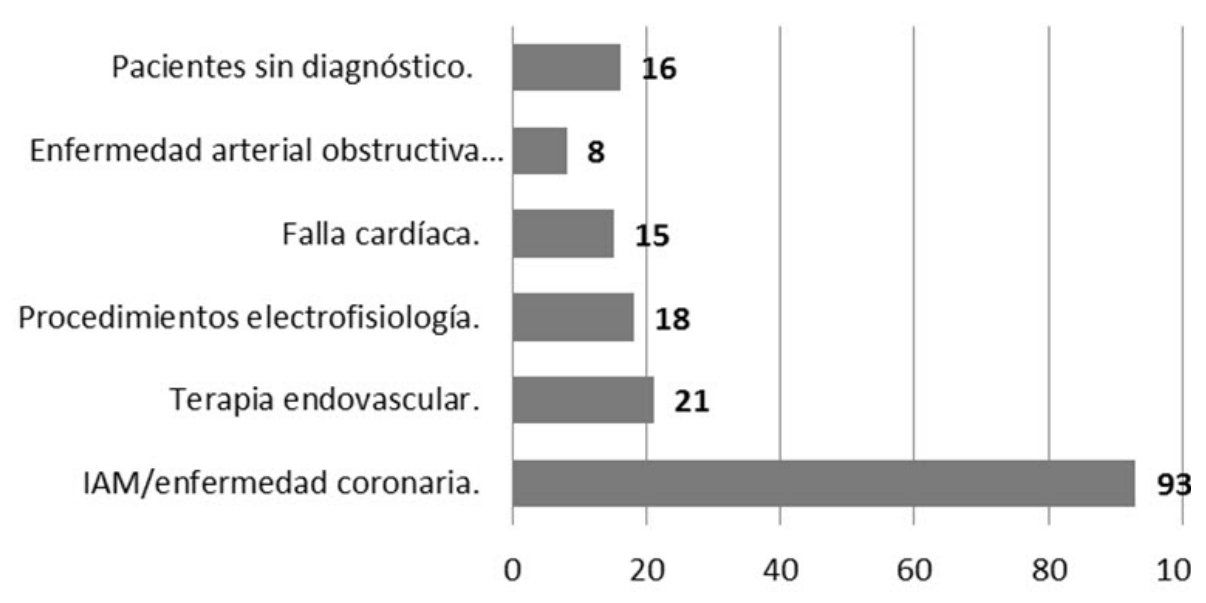

Figura 1. Primeros cinco diagnósticos de los pacientes hospitalizados en UCC 


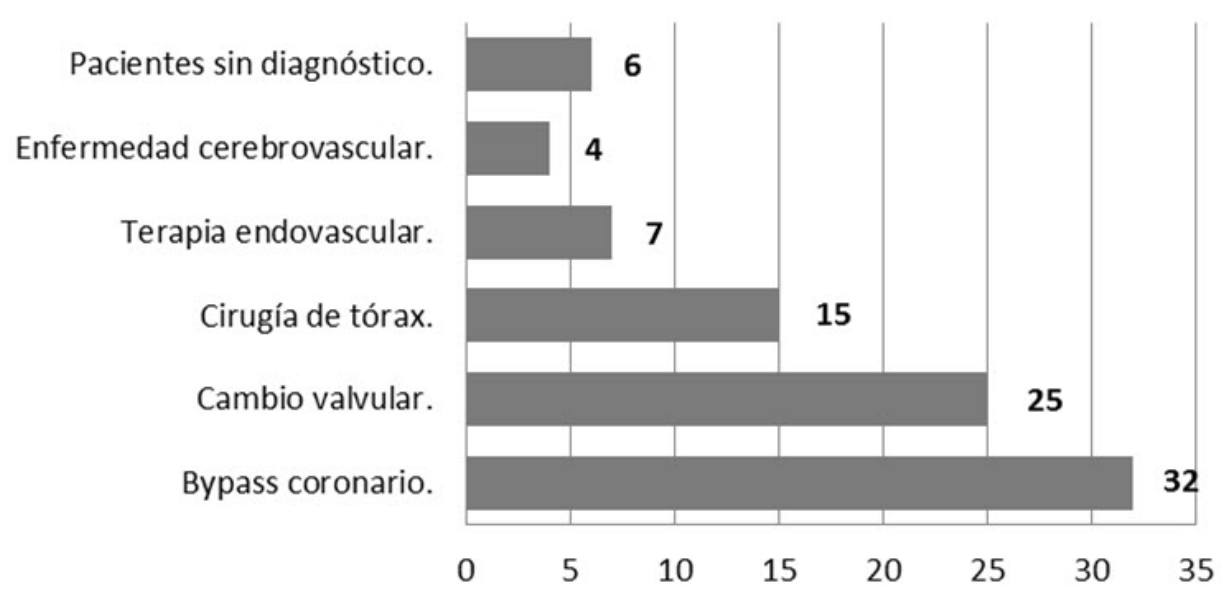

Figura 2. Primeros cinco diagnósticos de los pacientes hospitalizados en UCI

Tabla 2. Parentesco de personas encuestadas

\begin{tabular}{lc}
\hline \multicolumn{1}{c}{ Parentesco } & $\%$ \\
\hline Esposa/o & 32,5 \\
Hijo/a & 37,9 \\
Hermano/a & 11,9 \\
Padre & 0,6 \\
Madre & 3,9 \\
Amigo/a & 2,3 \\
Cuñada & 7,1 \\
Paciente & 3,2 \\
Sobrino/a & 0,6 \\
\hline
\end{tabular}

Fuente: Datos del estudio
De los familiares entrevistados, el $75 \%$ informaron que su parentesco con el paciente era esposo/a, hijo/a y hermano/a. (Tabla 2).

Con respecto a la información brindada sobre el estado del paciente y la terminología utilizada, 231 de los encuestados (73,8 \%) respondieron que casi siempre se utilizaron palabras que podían entender, 230 (73,2 \%) entendían lo que le ocurría la mayor parte del tiempo a su familiar y los medios o equipos médicos que se estaban utilizando para su curación, 233 (74 \%) percibieron que la información brindada acerca del paciente fue sincera pero solo el 50.3 \% (157 encuestados) afirmaron que recibieron información sobre los equipos médicos que se estaban utilizando en su familiar y el 16,7 \% (52 personas) nunca recibieron dicha información.

A las preguntas relacionadas con los cuidados recibidos por el paciente, 232 (73,9\%) consideraron que el cuidado por parte del personal sanitario (médicos, enfermeras, auxiliares) fue adecuado, el 56,9 \% (174 encuestados) perciben que los cuidados médicos recibidos no pueden ser mejores y el $15 \%$ (46 encuestados) piensan que si pueden serlo. A la pregunta usted cree que a su familiar, en este hospital, se le están dando los mejores cuidados posibles, o cree que serían mejores en otro hospital el $41,7 \%$ (129 encuestas) responden casi siempre y el $39.2 \%$ nunca.

Sobre la amabilidad percibida por los familiares, 233 personas $(73,7 \%)$ consideran que el trato por parte del personal médico fue amable y 79 (25\%) consideran que fueron amables muchas de las veces. Los médicos preguntaron por necesidades especiales a los acompañantes en el $36.8 \%$ de las encuestas (116) y nunca preguntaron al 29,8 \% (94). 199 encuestados (63,6 \%) consideran que en caso de alguna urgencia le llamaran a su casa aunque $11,5 \%$ (36) consideran que no.

Con respecto a la comodidad durante la estancia en la unidad, 229 (73,2 \%) se sienten cómodos durante la visita, 58,9 \% (182) piensan que la sala de espera es confortable y 66,6 \% (209) nunca se sintieron solos o aislados en la sala de espera.

Los resultados satisfactorios globales de la encuesta se encuentran en la tabla 3 y figura 3. 
Los resultados expuestos en la tabla 4 deben ser analizados para proponer opciones de mejora, ya que las explicaciones sobre los equipos médicos utilizados, la percepción de los cuidados recibidos por el paciente, las preguntas hechas por los médicos a las familias y acompañantes sobre sus necesidades y la confianza de que los llamaran a su casa en caso de alguna urgencia médica obtuvieron respuestas no siempre satisfactorias y que una vez mejoradas pueden generar mucha más empatía con el personal médico en momentos tan críticos y además garantizar la tranquilidad y seguridad de que a su familiar se le estén dando los mejores cuidados posibles.

Tabla 3. Resultados satisfactorios globales y por servicio de la encuesta de satisfacción

\begin{tabular}{|c|c|c|c|}
\hline Pregunta & UCI & UCC & Global \\
\hline Comprensión de lo que le ocurría la mayor parte del tiempo a su familiar & $75,5 \%(1)$ & $72,1 \%(1)$ & $73,2 \%(1)$ \\
\hline $\begin{array}{l}\text { Información brindada sobre el estado del paciente y la terminología } \\
\text { utilizada }\end{array}$ & $78,5 \%(1)$ & $71,4 \%(1)$ & $73,8 \%(1)$ \\
\hline Sinceridad de la información brindada acerca de la condición del paciente & $79,4 \%(1)$ & $71,2 \%(1)$ & $74,0 \%(1)$ \\
\hline Percepción de cuidado del paciente por parte del personal sanitario. & $79,4 \%(1)$ & $71,0 \%(1)$ & $73,9 \%(1)$ \\
\hline Sobre la amabilidad del personal percibida por los familiares & $82,2 \%(1)$ & $70,0 \%(1)$ & $74,2 \%(1)$ \\
\hline Comodidad de los acompañantes durante la visita & $75,7 \%(1)$ & $71,8 \%(1)$ & $73,2 \%(1)$ \\
\hline Soledad y aislamiento en la sala de espera. & $70,4 \%(2)$ & $64,6 \%(2)$ & $66,6 \%(2)$ \\
\hline
\end{tabular}

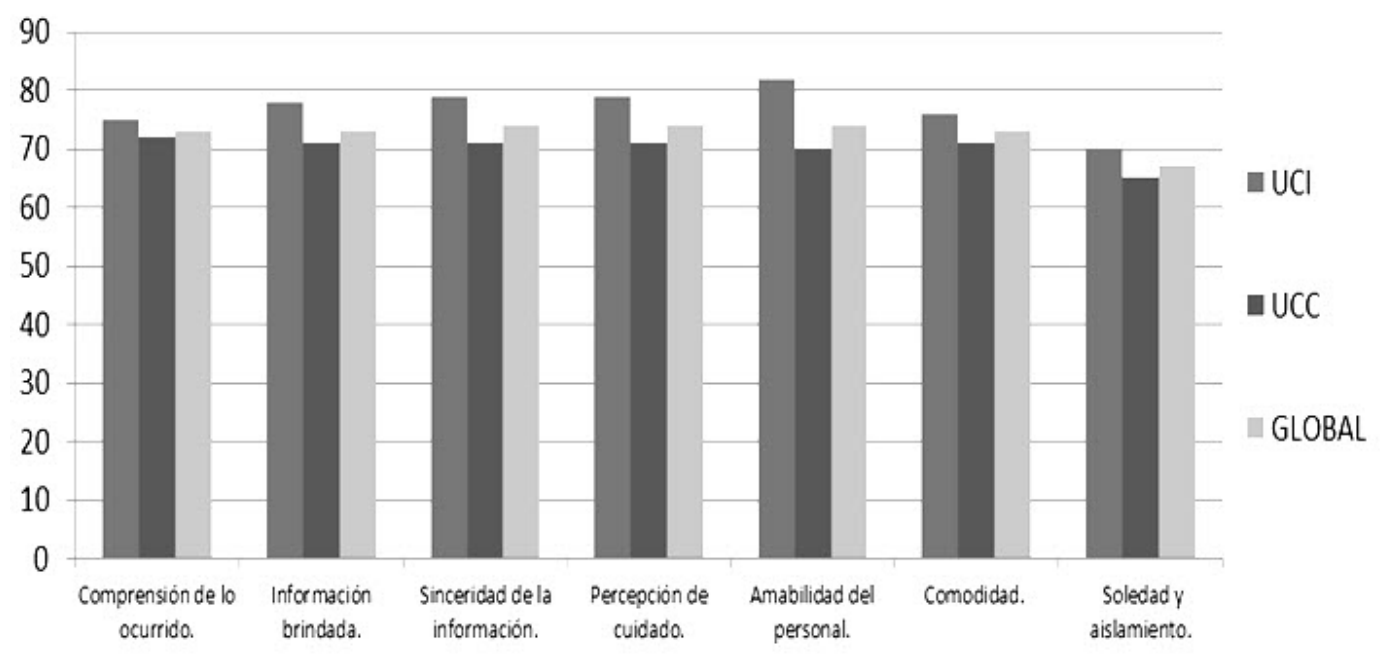

Figura 3. Resultados satisfactorios globales y por servicio de la encuesta de satisfacción 
Tabla 4. Resultados por unidad y en forma global de la Institución

\begin{tabular}{lccc}
\hline \multicolumn{1}{c}{ Pregunta } & UCI & UCC & Global \\
\hline $\begin{array}{l}\text { Explicación de los medios o equipos médicos que se estaban } \\
\text { utilizando para la curación de su familiar. }\end{array}$ & $47.6 \%(1)$ & $51.7 \%(1)$ & $50.3 \%(1)$ \\
\hline Considera que algunos de los cuidados médicos recibidos por el & $17.1 \%(2)$ & $16,4 \%(2)$ & $16.7 \%(2)$ \\
paciente pueden ser mejores & $64.8 \%(2)$ & $52.7 \%(2)$ & $56.9 \%(2)$ \\
\hline Cree que a su familiar se le están dando los mejores cuidados & $33.3 \%(1)$ & $45,1 \%(1)$ & $41.7 \%(1)$ \\
posibles, o cree que serían mejores en otro hospital & $51.5 \%(2)$ & $33 \%(2)$ & $39.2 \%(2)$ \\
\hline $\begin{array}{l}\text { Preguntas de los médicos por necesidades especiales a los } \\
\text { acompañantes. }\end{array}$ & $39.8 \%(1)$ & $35.3 \%(1)$ & $63.6 \%(1)$ \\
\hline En caso de alguna urgencia considera le llamaran a su casa & $25.9 \%(2)$ & $31.9 \%(2)$ & $11.5 \%(2)$ \\
\hline La sala de espera es confortable & $71.8 \%(1)$ & $57.6 \%(1)$ & $63.6 \%(1)$ \\
\hline
\end{tabular}

$1=$ Casi siempre, $2=$ Nunca, $3=$ Algunas veces, $4=$ Muchas de las veces. Fuente: Datos del estudio

\section{Discusión}

La satisfacción de los familiares de los pacientes que son ingresados a la unidad de cuidado intensivo o cuidado coronario debe ser tenida en cuenta como un atributo de calidad y por lo tanto debe medirse. De acuerdo a sus resultados se debe definir la necesidad de implementar acciones de mejora.

La encuesta utilizada en el presente estudio fue validada y publicada en España y evalúa los siguientes aspectos: la información brindada a los acompañantes y la comprensión del estado del familiar, los percepción de los cuidados recibidos por el paciente, la amabilidad del personal hacia el paciente y sus acompañantes y la comodidad durante la estancia en la unidad.

En las unidades evaluadas se encontró que la mayoría de las personas encuestadas consideran que la información acerca del estado de su familiar fue adecuada, la terminología fue clara y comprendían lo que le estaba ocurriendo al paciente. Un alto porcentaje de personas no recibieron suficiente información sobre los equipos y los medios utilizados en el paciente e incluso algunos nunca recibieron información. La comunicación efectiva tanto con el paciente como con su familia puede generar tranquilidad y seguridad y hacer que la estancia sea menos estresante mejorando el bienestar psicológico y mental de ambos actores y favoreciendo la recuperación (24). La percepción de los cuidados del paciente por parte del personal médico fue muy buena.

En cuanto a la amabilidad del personal, los encuestados tienen una buena percepción de la misma aunque muchos afirman que el personal médico nunca pregunto por sus necesidades como acompañantes y en la unidad coronaria un alto porcentaje de personas consideran que no se les llamara a su casa en caso de que algo le ocurra a su familiar. Esta descrito que el personal médico y de enfermería en ocasiones falla en tratar de indagar por las necesidades de los miembros de la familia (3) aunque otros a su vez comentan que el personal de enfermería tiene mayor capacidad de entablar una mejor relación con la familia del paciente que el mismo médico, durante el desarrollo de las actividades diarias $(\underline{25}, \underline{26})$. 
Sería relevante indagar además por las necesidades del personal médico y de enfermería frente al paciente y a su familia lo que puede contribuir a mejorar la relación de todo el personal con los pacientes y familiares (27-29).

Los acompañantes de los pacientes de UCl perciben la sala de espera como menos confortable que los de la UCC pero se sienten menos solos y aislados en la UCl.

El tipo de respuestas de la encuesta casi siempre, muchas de las veces, algunas veces o nunca no responden a todas las posibilidades para conocer la satisfacción de un paciente o su acompañante, ya que no se incluyó la posibilidad de calificar siempre a alguna de las preguntas.

Los resultados a la pregunta ¿Usted cree que a su familiar, en este hospital, se le están dando los mejores cuidados posibles, o cree que serían mejores en otro hospital? deben analizarse ya que dicho enunciado contempla dos posibilidades, la primera es que se considere que el paciente recibe los mejores cuidados posibles en la institución y la otra es que se considere que dichos cuidados sean mejores en otro hospital; ya que no hay claridad en la pregunta, la respuesta tampoco lo es, obteniendo resultados no concluyentes.

Los resultados de la encuesta muestran una tendencia hacia la satisfacción de los acompañantes aunque hay puntos que se deben evaluar en la institución como son las necesidades especiales a los acompañantes, la explicación de los medios o equipos que se utilizan en el paciente y la confianza de los acompañantes de que se les llamara en caso de una urgencia dada la gravedad de los pacientes atendidos en dichos servicio.

\section{Bibliografía}

1. Simon VJ. Desarrollo de un procedimiento para la medición de la satisfacción del cliente en una industria auxiliar del sector carrocero de autocares y autobuses: Estudio la satisfacción del cliente. Trabajo de investigación de la Universidad de Sevilla [Acceso 2014 Marzo 22. Disponible en: http://bibing.us.es/proyectos/ abreproy/3966/fichero/1 \%252F2.pdf.

2. Gómez S, Ballester A, Gil Ju. The short version of Critical Care Family Needs Inventory CCFNI adaptation and validation for a spanish sample. An Sist Sanit Navar. 2011 Sep-Dec;34(3):349-61. http://www.ncbi.nlm.nih.gov/pubmed/22233839

3. Verhaeghe S, Defloor T, Van Zuuren F, Duijnstee M, Grypdonck M. The needs and experiences of family members of adult patients inan intensive care unit: a review of the literature. J Clin Nurs. 2005 Apr;14(4):501-9. http://www.ncbi.nlm.nih. gov/pubmed/15807758

4. Susan M. Roberti and Joyce J. Fitzpatrick. Assessing Family Satisfaction With Care of Critically III Patients: A Pilot study. Crit Care Nurse 2010;30:18-26. http:// www.ncbi.nlm.nih.gov/pubmed/21123230

5. Gillis CL. Reducing family stress during and after coronary artery bypass surgery.Nurs Clin North Am 1984; 19: 103-112. http://www.ncbi.nlm.nih.gov/pubmed/6608097 
6. Leske JS. Needs of relatives of critically ill patients: a followup. Heart and Lung 1986; 189-193. http://www.ncbi.nlm.nih.gov/pubmed/3633247

7. Leske JS. Internal psychometric properties of the Critical Care Family Needs Inventory. Heart Lung 1991; 20: 236-344. http://www.ncbi.nlm.nih.gov/pub$\underline{\mathrm{med} / 2032860}$

8. Harvey M. Volunteers in the critical care waiting room. Anaheim, CA: Society of Critical Care Medicine 1993; 79-80. http://www.atsjournals.org/doi/full/10.1164/ ajrccm.163.1.2005117\#.V-LXVPI97IU

9. Johnson D, Wilson M, Cavanaugh B, Bryden C, Gudmundson D, Moodley O. Measuring the ability to meet family needs in an intensive care unit. Crit Care Med 1998; 26: 266-271. http://www.ncbi.nlm.nih.gov/pubmed/9468163

10. Anales del Sistema Sanitario de Navarra versión impresa ISSN 1137-6627 Anales Sis San navarra vol.34 no.3 Pamplona set.-dic. 2011

11. Mendonca D, Warren NA. Perceived and unmet needs of critical care family members. Crit Care Nurs Q 1998; 21: 58-67. http://www.ncbi.nlm.nih.gov/pubmed/9644362

12. Azoulay E, Pochard F, Chrevret S, LeMaire, F, Mokhtari M, LeGall J, et al. Meeting the needs of intensive care unit patient families. Am J Respir Crit Care Med. 2001;163:135-139. http://www.ncbi.nlm.nih.gov/pubmed/11208638

13. Koenig HG, George LK, Stangl D, Tweed DL. Hospital stressors experienced by elderly medical patients: developing a Hospital Stress Index. Int J Psychiatry Med 1995; 25: 103-122. http://www.ncbi.nlm.nih.gov/pubmed/7649715

14. Hampe 1975, Daley 1984, Lynn-McHale \& Bellinger 1988, Forrester et al. 1990, Jacono et al. 1990, Kleinpell \& Powers 1992, Warren 1993, Mendonca \& Warren 1998

15. Molter 1979, O'Neill Norris \& Grove 1986, Lynn-McHale \& Bellinger 1988, Forrester et al. 1990, O'Malley et al. 1991, Kleinpell \& Powers 1992, Murphy et al. 1992, Bijttebier et al. 2001, Delva et al. 2002).

16. Hickey M: What are the needs of families of critically ill patients? A review of the literature since 1976. Heart Lung 1990; 19:401-415. http://www.ncbi.nlm.nih. gov/pubmed/2196246

17. Molter NC: Needs of relatives of critically ill patients: a descriptive study. Heart Lung 1979; 8:332-339). http://www.ncbi.nlm.nih.gov/pubmed/253712

18. Kirchhoff KT, Walker L, Hutton A, et al: The vortex: Families' experiences with death in the intensive care unit. Am J Crit Care 2002; 200-209 http://www.ncbi. nlm.nih.gov/pubmed/12022483

19. Truog RD, Cist AFM, Brackett SE, et al: Recommendations for end-of-life care in the intensive care unit: The Ethics Committee of the Society of Critical Care Medicine. Crit Care Med 2001; 29:2332-2347 http://www.hadassah-med.com/medi a/1884680/15recomrecomendations.pdf 
20. Clarke EB, Curtis JR, Luce JM, et al: Quality indicators for end-of-life care in the intensive care unit. Crit Care Med 2003; 31:2255-2262 http://www.ncbi.nlm.nih. gov/pubmed/14501954

21. Torrents R, Oliva E, Saucedo MJ, Surroca L, Jover C. Impacto de los familiares del paciente crítico ante una acogida protocolizada. Enferm intensiva 2003; 14 : 49-60. http://www.sciencedirect.com/science/article/pii/S1130239903781048

22. Vincent JL. Communication in the ICU. . Intensive Care Med, 23 (1997), pp. 1093-8 http://link.springer.com/article/10.1007\%2Fs001340050462?LI=true

23. Kleinpell RM, Powers MJ: Needs of family members of intensive care unit patients. Appl Nurs Res, 5 (1992), pp 2-8. http://www.ncbi.nlm.nih.gov/pubmed/1570953

24. Hupcey JE. Establishing the nurse - family relationship in the intensive care unit. http://www.ncbi.nlm.nih.gov/pubmed/9550930

25. Novaes MAF.P,Knobel E,Bork AM,Pavao OF,Nogueira-Martins LA, Ferraz MB. Stressors in ICU: perception of the patient, relatives and health care team. Intensive Care Med, 25 (1999), pp. 1421-6 http://www.ncbi.nlm.nih.gov/pubmed/10660851

26. Bijttebier P,Vanoost S,Delva D,Ferdinande P,Frans E. Needs of relatives of critical care patients: perceptions of relatives, physicians and nurses. Intensive Care Med, 27 (2001), pp. 160-5 http://www.ncbi.nlm.nih.gov/pubmed/11280629

27. Myhren H,Ekeberg O,Langen I,Stokland O. Emotional strain, communication, and satisfaction of family members in the intensive care unit compared with expectations of the medical staff: experiences from a Norwegian University Hospital. Intensive Care Med, 30 (2004), pp. 1791-8 http://www.ncbi.nlm.nih.gov/pubmed/15258729 